\title{
Downlink beamforming concepts in UTRA FDD
}

\author{
M. Schacht ${ }^{1}$, A. Dekorsy ${ }^{1}$, and P. Jung ${ }^{2}$ \\ ${ }^{1}$ Lucent Technologies, Thurn-und-Taxis-Strasse 10, 90411 Nuremberg, Germany \\ ${ }^{2}$ Gerhard-Mercator-Universität Duisburg, Oststrasse 99, 47057 Duisburg, Germany
}

\begin{abstract}
This article gives a comparison of beamforming concepts. Adaptive beamforming and fixed beam switching in WCDMA-FDD-mode are compared from a system level perspective, ordinary sectorization (three $120^{\circ}$ sectors) serves as a basis for comparison. Pilot channels P-CPICH (Primary Common Pilot Channel) and S-CPICH (Secondary $\mathrm{CPICH}$ ) are considered as additional interference. For adaptive beamforming channel estimation has to be based on the pilot bit sequence on DPCCH (Dedicated Physical Control Channel) which leads to degradation especially for high mobile velocities and large angular dispersions of the multipath channel.
\end{abstract}

\section{Introduction}

In 3rd generation mobile communication systems, and especially in UMTS (Universal Mobile Telecommunication System), exploitation of the spatial dimension of the mobile channel promises great benefits. In order to establish spatial filters antenna arrays are used and beamforming concepts are evaluated. The concept of adaptive beamforming is a sophisticated but complex way to exploit the spatial dimension of the mobile radio channel. For every user in the CDMAsystem an individual beam pattern is formed and adapted to the particular network condition. A drawback of this concept, however, is the downlink channel estimation. While for regular sector cells a common pilot channel, the primary common pilot channel (P-CPICH), serves as phase reference for channel estimation, this channel cannot be used when adaptive beamforming is applied, since every other user has a different beampattern and therewith phase reference. Provision of user-specific pilot channels would cause too much inference in the system, thus channel estimation has to be based on the pilot bits on the dedicated physical control channel (DPCCH) and a link degradation has to be accepted (app. $3 \mathrm{~dB}$ ). Alternatively beam-switching concepts are evaluated,

Correspondence to: M. Schacht (mschacht@lucent.com) i.e. a certain number of fixed beams is provided, each having an individual pilot channel, so-called secondary common pilot channels (S-CPICH) for beam specific channel estimation. The drawback, of course, is that the beams are fixed and degrade especially in environments with large angular spreads of the incoming user signals. The new aspect of this paper is the comparison of the two concepts on a basis of system level simulation results for downlink transmission in UTRA-FDD (UMTS Terrestrial Radio Access). The paper is structured in four sections. After general thoughts about beamforming (Sect. 2.1) the concepts of beam switching (Sect. 2.2) and adaptive beamforming (Sect. 2.3) are introduced. A system-level simulator is described in Sect. 3 and in Sect. 4, simulation results are discussed. The paper is concluded in Sect. 5.

\section{Concept descriptions}

\subsection{General thoughts}

The investigations and simulations that are done for this paper refer to a UMTS cellular network. Only the UTRA FDD mode is examined. Standard hexagonal cells are divided into three $120^{\circ}$ sectors, each operating a uniform linear antenna array (ULA) consisting of four identical antenna elements with $120^{\circ}$ HPBW (Half Power Beam Width); an interelement spacing of $d=\frac{\lambda}{2}$ is assumed, $\lambda$ corresponds to the wavelength of the incident waves. With this assumption the steering vector for a four element ULA results in

$\mathbf{a}(\phi)=\left(1, e^{-j \pi \sin \phi}, e^{-j 2 \pi \sin \phi}, e^{-j 4 \pi \sin \phi}\right)^{T}$,

where $\phi$ is the azimuth angle and $T$ indicates the transpose. Note that beamforming concepts examined here are applied to dedicated channels (DCHs), while broadcast channels (broadcast channels, primary pilot channels etc.) are transmitted via a single sector antenna beampattern, compare Figs. 1 and 2. 


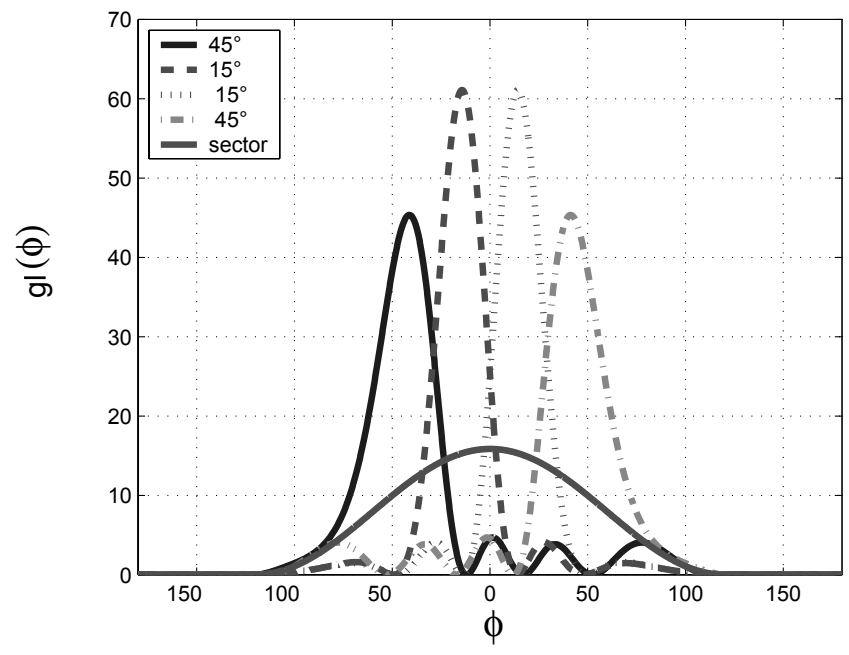

Fig. 1. Different beampatterns formed in beam switching mode within each sector, downlink. Beampattern of each individual antenna with $120^{\circ} \mathrm{HPBW}\left(g_{\text {sec }}(\phi)\right.$ corresponds to "sector").

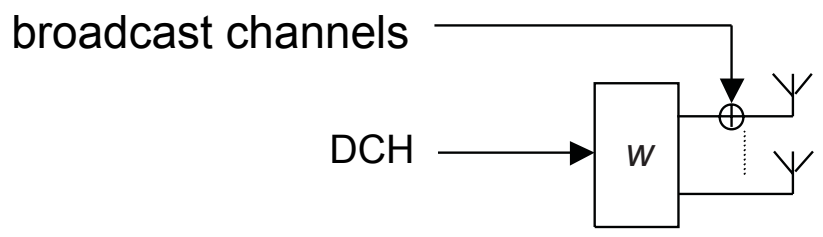

Fig. 2. Broadcast channels and $\mathrm{DCH}$, w represents the complex weighting/beamforming vector.

\subsection{Fixed beam switching}

For the fixed beam switching case, four fixed beams per sector are formed, e.g. using a Butler matrix (Litva and Kwok-Yeung Lo, 1996). The angles $\phi_{l}, l \in$ [1..4] of the main directions for the four beams per sector are set to $\phi_{l} \in\left[-45^{\circ},-15^{\circ}, 15^{\circ}, 45^{\circ}\right]$ with regard to the center axis of a sector, see Fig. 1. The weighting vectors $\mathbf{w}_{l}, l \in[1 . .4]$, of the beams are applied to the according steering vectors, $\mathbf{w}_{l}=\frac{1}{2} \mathbf{a}\left(\phi_{l}\right), l \in[1 . .4]$. The amplitude factor of $\frac{1}{2}$ assures that the transmit power is distributed equally to the four antenna elements (downlink direction is investigated). With the beampattern $g_{s e c}(\phi)\left(120^{\circ} \mathrm{HPBW}\right)$ of the individual antenna, the total beampatterns result in

$$
\begin{aligned}
g_{l}(\phi) & =\left|\mathbf{w}_{l}^{H} \mathbf{a}(\phi)\right|^{2} g_{s e c}(\phi) \\
& =\frac{1}{4}\left|\mathbf{a}\left(\phi_{l}\right)^{H} \mathbf{a}(\phi)\right|^{2} g_{s e c}(\phi), \quad l \in[1 . .4]
\end{aligned}
$$

and are displayed in Fig. 1. For beam assignment of the mobiles uplink measurements are evaluated, i.e. the beam with highest power in the uplink is used for downlink transmission, compare Technical Report TSGR 25.887 (2001). To enable proper channel estimation and beam distinction ability secondary common pilot channels (S-CPICH) are provided within each beam. UMTS-systems actually use the
Primary CPICH for channel estimation when run in sectorized mode (cells divided in three sectors, no beamforming), since beampatterns for P-CPICH and dedicated channels are identical. In beam switching mode though, P-CPICH uses the sector pattern while dedicated channels use the beampatterns, therefore additional S-CPICHs are proposed to be implemented, compare Technical Report TSGR1-23 (2002). For the power setting of the S-CPICH it is here assumed that users should experience the same pilot-to-interference-ratio for the S-CPICH as for the P-CPICH. So

$\frac{P_{P-C P I C H}}{I_{P-C P I C H}}=\frac{P_{S-C P I C H}}{I_{S-C P I C H}}$.

Since the S-CPICH uses the beampatterns, its antenna gain is higher than that of the P-CPICH, approximately by the number of antenna elements used to form the fixed beams, compare Fig. 1. Due to the spatial separation of the interferers the beam interference reduces also approximately by the number of beams (i.e. it is assumed that the coverage area of a beam is the sector coverage area uniformly distributed to the beams). Thus S-CPICH power is set $12 \mathrm{~dB}$ below P-CPICH. Note that there are other suggestions on the power relations between P-CPICH and S-CPICH within 3GPP, see Technical Report TSGR1-23 (2002). Figure 3 shows an example of a fixed beamswichting scenario. The user path is indicated by the solid line, the interferer's by the dashed line. The diagram shows the path power including the antenna gain, i.e. backward paths are suppressed.

\subsection{User specific beamforming (adaptive beamforming)}

To establish an adaptive beamforming concept, spatial information about the individual user has to be retrieved and processed. From uplink transmission spatial covariance matrices are obtained, since only the long term statistic is evaluated the uplink/downlink transformation is valid. The following assumptions are made, compare Czylwik and Dekorsy (2001):

- A user $\mathrm{k}$ is assigned to a single sector in the system, hand over issues are neglected.

- Between each base station (BS) and every user $k$ there is a finite set of paths $N_{k}$, which follows a binomial distribution between 3 and a maximum of 6 paths.

- The DOAs (directions of arrival) $\theta(i, k)$ as well as the path coefficients $h(i, k)$ (path $i$ of user $k$ ) are perfectly estimated; $h(i, k)$ represents pathloss and fading (lognormal as well as Rayleigh fading).

- All user signals are transmitted with the same power $P$, power control is neglected.

- The optimal downlink beampatterns can be obtained from the uplink covariance matrices (long term statistic is exploited), downlink broadcast channels have to be taken into account. 
For each user $k$ assigned to a certain sector, two spatial covariance matrices are distinguished, one for the desired signal, noted as $\mathbf{R}_{k}^{S}$ and another gathering the interfering signals $\mathbf{R}_{k}^{I}$ :

$\mathbf{R}_{k}^{S}=\sum_{i=1}^{N_{k}} \mathbf{a}\left(\theta_{(i, k)}\right) \mathbf{a}^{H}\left(\theta_{(i, k)}\right) P E\left\{\left|h_{(i, k)}\right|^{2}\right\}$,

$\mathbf{R}_{k}^{I}=\sum_{\substack{j=1 \\ j \neq k}}^{N_{m o b}} \sum_{i=1}^{N_{j}} \mathbf{a}\left(\theta_{(i, j)}\right) \mathbf{a}^{H}\left(\theta_{(i, j)}\right) P E\left\{\left|h_{(i, j)}\right|^{2}\right\}+\mathbf{I}_{N} P_{b c}$,

$N_{m o b}$ is the total number of users in the network, $H$ represents the hermitian transpose, $E\{$.$\} indicates the expected$ value (refers to Rayleigh fading), $I_{N}$ is a $N \times N$ identity matrix (here $N=4$ ) and $P_{b c}$ is the power of the downlink broadcast channels and thermal noise. Note that $E\left\{\left|h_{(i, k)}\right|^{2}\right\}$ represents the shadow fading. Rayleigh fading is considered analytically see Czylwik and Dekorsy (2001). The downlink broadcast channels as well as the thermal noise are modelled as additional spatially white noise. Therefore they add to the interference covariance matrix as a diagonal matrix with elements $P_{b c}$. Former evaluations, see Czylwik and Dekorsy (2001), only considered interference stemming from other users. Note that except for $P_{b c}$ the covariance matrices refer to the uplink. According to $\mathbf{R}_{k}^{S}$ and $\mathbf{R}_{k}^{I}$ the received power for the desired user is

$P_{k}^{S}=\mathbf{w}_{k}^{H} \mathbf{R}_{k}^{S} \mathbf{w}_{k}$

and respectively the interference power

$P_{k}^{I}=\mathbf{w}_{k}^{H} \mathbf{R}_{k}^{I} \mathbf{w}_{k}$.

Mathematically an optimization problem is obtained. The vector $\mathbf{w}_{k}, k \in\left[1, N_{m o b}\right]$ has to be optimized to obtain the maximum SINR. The problem is solved by applying the method of Lagrange multipliers, see Litva and Kwok-Yeung Lo (1996). The optimal weighting vector $\mathbf{w}_{\text {opt }}$ is the eigenvector that corresponds to the largest eigenvalue of the generalized eigenvalue problem

$\mathbf{R}_{k}^{S} \mathbf{w}=\lambda \mathbf{R}_{k}^{I} \mathbf{w}$

for the optimal beampattern yields

$g(\theta)=\left|\mathbf{w}_{o p t}^{H} \mathbf{a}(\theta)\right|^{2} g_{s e c}(\theta)$.

An example for an adaptively formed beampattern can be seen in Fig. 4. The interfering paths are dashed, while the desired path is black; its length indicates path strength. The interfering path arriving from app. $150^{\circ}$ is nulled out, while the desired path around $90^{\circ}$ experiences full antenna gain, also compare to Fig. 3. As mentioned before the draw back of this concept is that due to user-specific antenna diagrams the channel estimation has to be based on the pilot bit sequence on the DPCCH (Dedicated Physical Control Channel), which experiences the very same beampattern. Since this sequence is shorter than that of the P-CPICH, a link degradation occurs (Technical Report TSGR4-17, 2001).

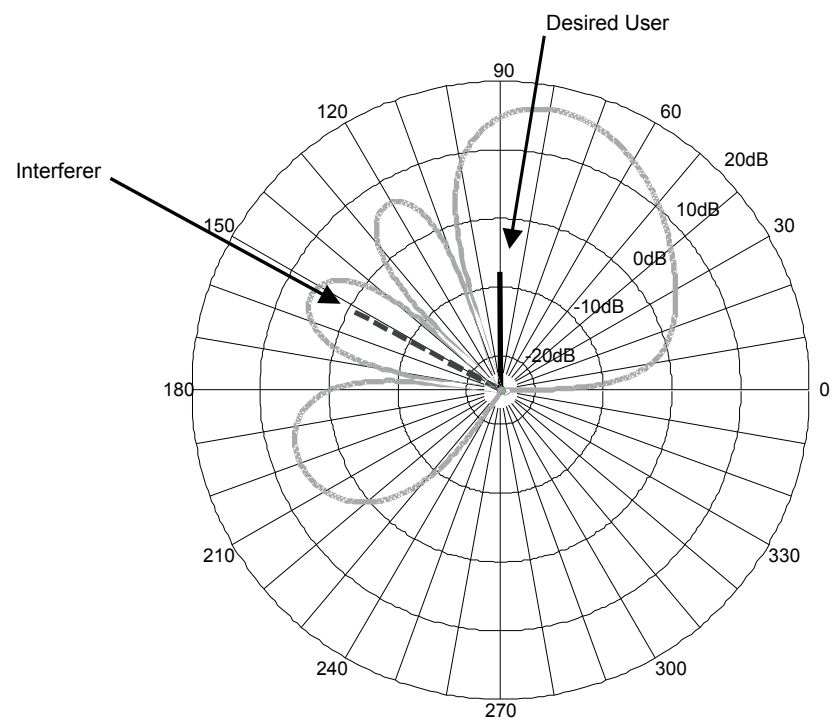

Fig. 3. Fixed beam swichting scenario.

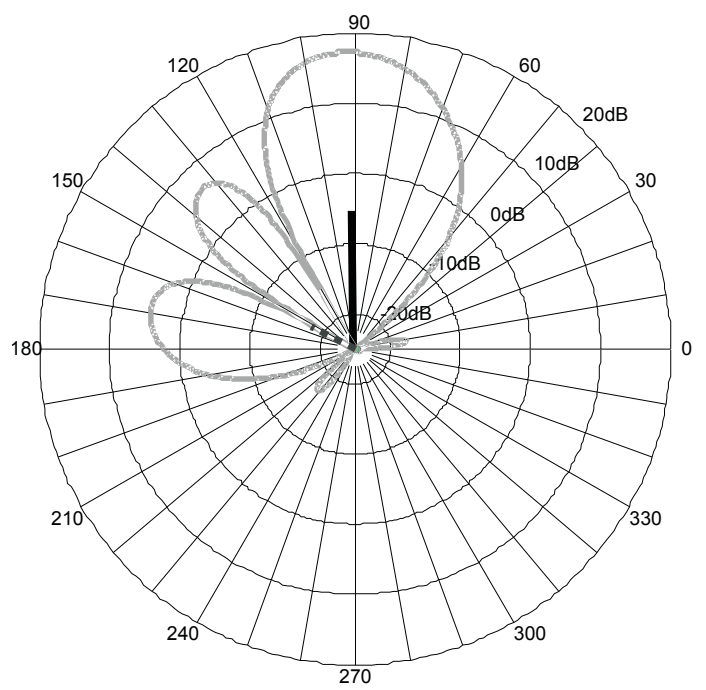

Fig. 4. Adapted beampattern.

\section{Simulator}

For this paper SINR distributions within cellular UMTS networks are investigated. Therefore a hexagonal cell structure is assumed and SINRs are calculated for mobiles within a set of centered reference cells, see Fig. 5. Each cell is divided into three sectors. Adaptive beamforming is done for every mobile in the network using one uniform linear array (ULA) per sector. Table 1 summarizes the important simulation parameters. Variables are

- Number of mobiles, i.e. network load

- Angular spread (AS) of the user signals 


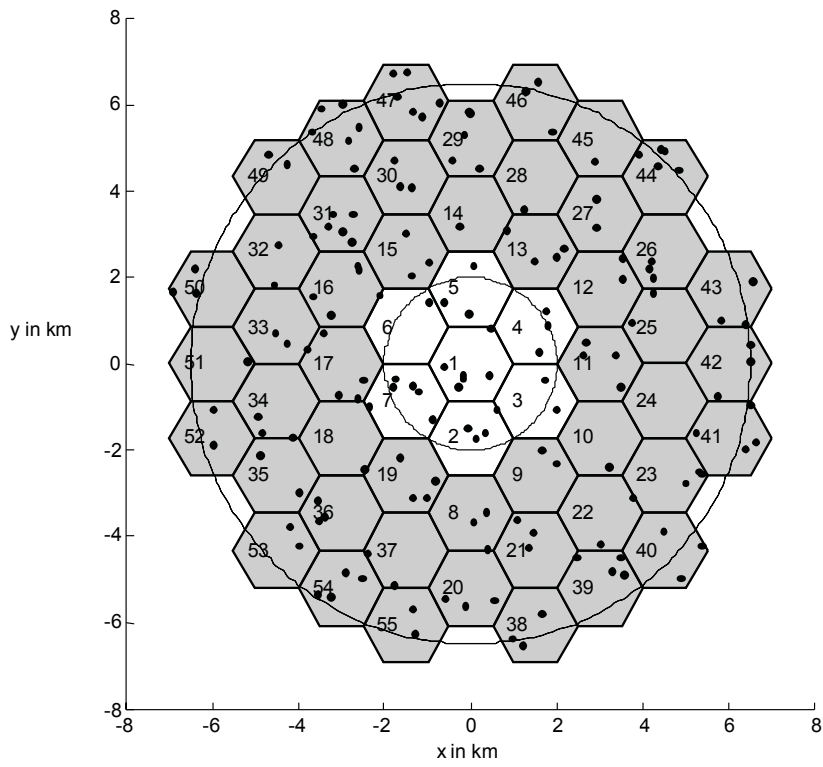

Fig. 5. Hexagonal network with reference cells.

Table 1. Table of simulation parameters

\begin{tabular}{l|l}
\hline ULA & 4 elements, $120^{\circ} \mathrm{HPBW}$ \\
Cell Radius & $1000 \mathrm{~m}$ \\
Thermal noise & $-102 \mathrm{dBm}$ \\
Lognormal fading & $8 \mathrm{~dB}$ (stand. dev.) \\
No. of paths & av. 3, max. 6 (binom. distr.) \\
Power per user(DCH) & $33 \mathrm{dBm}$ \\
Power of broadcast channel & $33 \mathrm{dBm}$ \\
Beam switching: & \\
S-CPICH power & $21 \mathrm{dBm}$ \\
Adap. beamforming: & \\
link degradation & $3 \mathrm{~dB}$ \\
& $($ Technical Report TSGR4-17, 2001) \\
No. of simulations & 100 \\
\hline
\end{tabular}

\section{Results}

First simulations were run for a network carrying a low load with 10 users per sector in average (maximum 20) and signals arriving at the base stations having small angular dispersions. For the angular spread a Laplacian distribution with zero mean and a variance of 1 degree around the main direction is assumed. As result, the averaged outage probability $P_{\text {out }}$ is displayed over the SINR. $P_{\text {out }}$ is the averaged CDF of the SINRs referring to mobiles in reference cells. Figure 6 clearly shows that for this configuration fixed beam switching and adaptive beamforming perform equally. Reason for this is the degradation of the channel estimation when using the adaptive concept (Technical Report TSGR4-17, 2001). This degradation was assumed to be $3 \mathrm{~dB}$. The actual degradation nevertheless depends on parameters like mobile speed and the number of pilot bits on the DPCCH. This issue is disregarded and for further examination. Note that for all

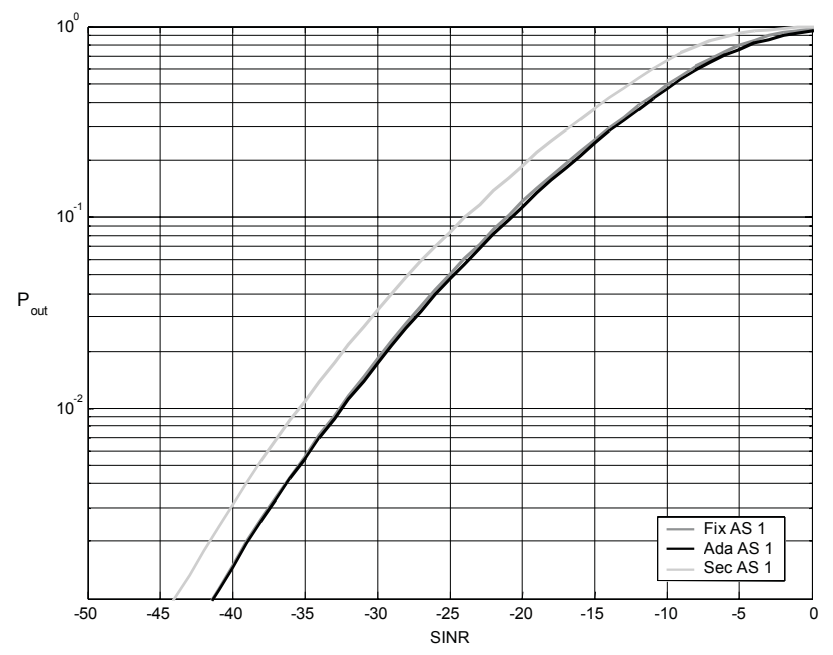

Fig. 6. Results for 10 users in average, $\max .20$ and $\mathrm{AS}=1^{\circ}$

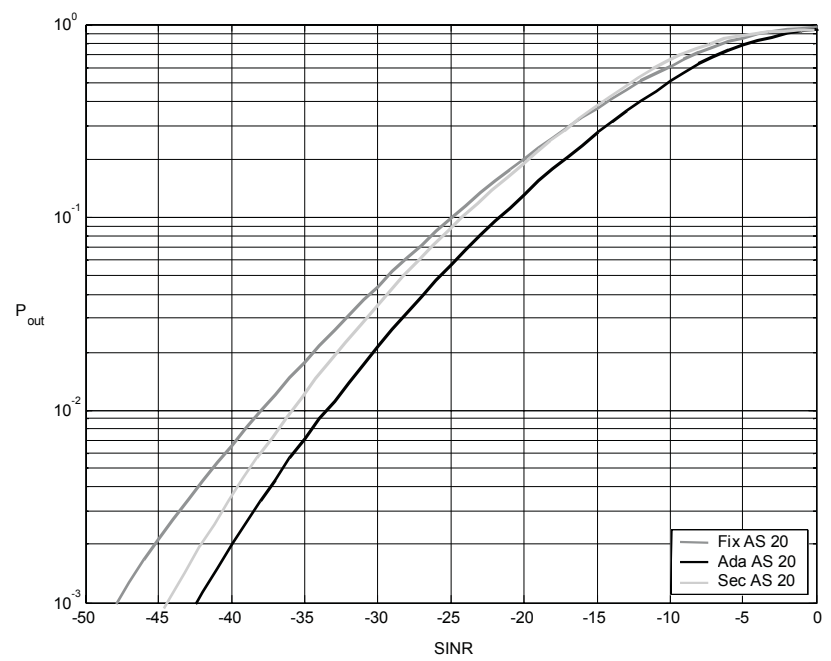

Fig. 7. Results for 10 users in average, $\max .20$ and $\mathrm{AS}=20^{\circ}$

investigations the link degradation when applying adaptive beamforming is assumed to be $3 \mathrm{~dB}$. Nevertheless both concepts clearly outperform the simpler sectorization concept.

Situation significantly changes when the angular dispersion of the incoming signals increases. For the next set of simulations the variance of the angular spread is set to 20 degrees. Figure 7 displays the superiority of the adaptive concept under these circumstances; the concept of fixed beam switching is inferior, even to ordinary sectorization. Figure 8 was found for a network with higher load, the number of users was increased to 30 in average and a maximum of 60. Therewith the influence of the S-CPICHs on the total interference was decreased. Figure 8 indicates that in network conditions with heavy loads and low angular spreads, the switched beam concept outperforms the adaptive. Finally Fig. 9 clearly shows, that for an angular dispersion of 20 degrees, the adaptive concept outperforms the fixed beam approach also for heavy load. 


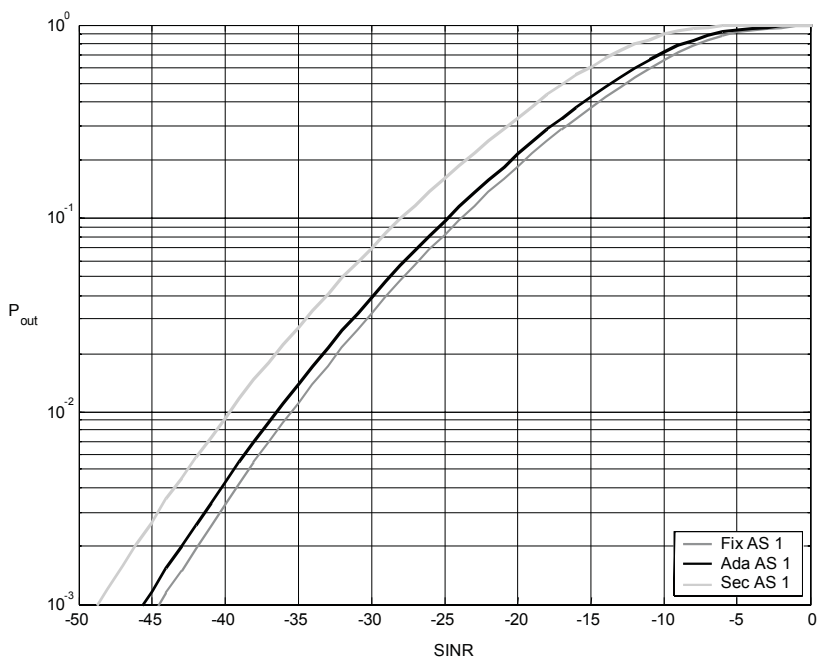

Fig. 8. Results for 30 users in average, $\max .60$ and $\mathrm{AS}=1^{\circ}$

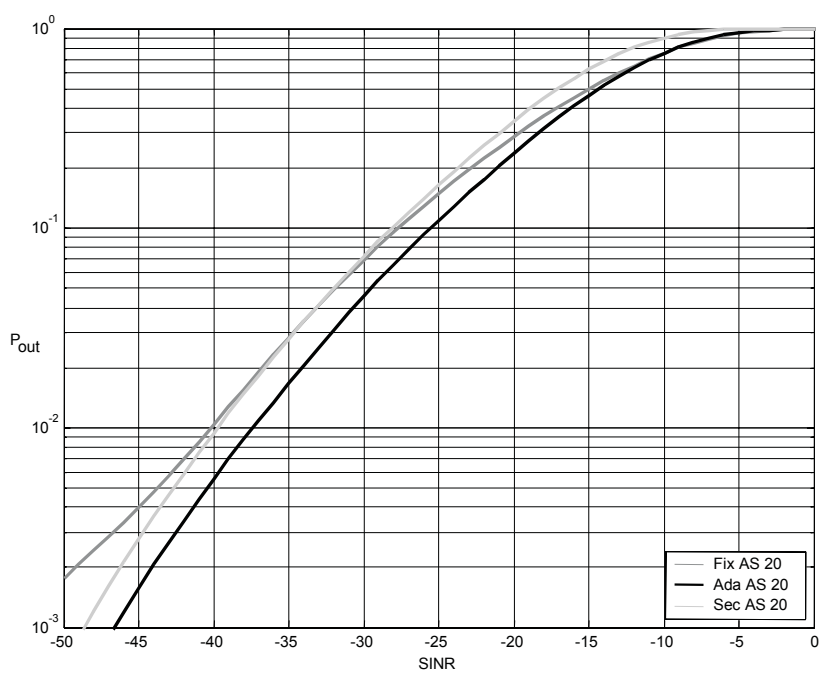

Fig. 9. Results for 30 users in average, $\max .60$ and $\mathrm{AS}=20^{\circ}$

\section{Conclusion}

Simulation results showed that compared to fixed beams, the adaptive concept degrades as the network load increases (for small angular spreads). As the number of interferers exceeds the number of antenna elements, the nulling advantage of the adaptive concept degrades, since suppression of all interferers is impossible. The adaptive algorithm then turns more and more into a beam steering concept, i.e. providing highest antenna gain to the desired user as the spatial interference becomes more and more white. Since a link degradation due to the channel estimation using DPCCH-bits has to be considered for the adaptive concept, the switched beam approach outperforms in high loaded scenarios for low angular dispersion. As the angular dispersion increases, the adaptive concepts is capable to adjust the beam direction and width, while the beam switching concept degrades. Note that for uplink beam switching, several beams can be exploited and "beam-diversity" gain can be obtained. For scenarios with high network loads and small angular spreads the switched beam concept should be preferred.

Acknowledgements. The authors would like to thank Andreas Czylwik for his contribution and his fundamental work on the simulator used for the investigations.

\section{References}

Litva, J. and Kwok-Yeung Lo, T.: Digital Beamforming in Wireless Communications, Artech House, Boston, 1 Edition, 1996.

Technical Specification Group Radio Access Network 3 GPP, Beamforming enhancements (release 5), Technical Report 25.887, ETSI, 2001.

Technical Specification Group Radio Access Network 3GPP, 3GPP Beamforming vs. Eigenbeamformer, Technical Report TSGR123, R1-02-0117, ETSI, 2002.

Czylwik, A. and Dekorsy, A.: System Level Simulations for Downlink Beamforming with Different Array Topologies, In Proc. IEEE Global Conference on Communications (GLOBECOM), San Antonio, USA, November 2001.

Technical Specification Group Radio Access Network 3 GPP, Proposal for user specific beamforming for UTRA FDD, Technical Report TSGR4-17, ETSI, 2001. 